Schweiz. Z. Tuberk. 1960;17:I-IV

\title{
Contents, Vol. 17, 1960
}

\section{INDEX}

Aguet, M.: $\quad$ vide Bonstein, $\mathrm{H}$.

Baly, Z.: $\quad$ vide Ostry, P.

Bertrab, R. von: $\quad$ vide Herzog, $\mathrm{H}$.

Bonstein, H.: vide Favez, G.

Bonstein, H. et Favez, G.:

Résultats compares de la Kanamycine et la streptomycine dans le traitement de la tuberculose pulmonaire

Bonstein, H.; Rossi, G. et Favez, G.:

Rapport entre Tinactivation individuelle de $\Gamma$ lsoniazide, les résultats bactériologiques et la regression des cavernes tuberculeuses ....

Bonstein, H.; Rossi, G.; Fried rich, T.; Lauener, H.; Aguet, M. et Favez, G.:

Influence de Tapport de Pyridoxine sur $\Gamma$ activité des transaminases

chez les malades traités par $\Gamma$ lsoniazide

Brunner, K.: Regionale Lymphknotenkomplikationen nach BCG-Impfung . . .

Burri, H.P.: vide Herzog, H.

Cardis, F.: $\quad$ La rechute dans la tuberculose pulmonaire de $\Gamma$ adulte: son importance

dans le recrutement de quelques sanatoria suisses en 1957 et 1958 .

Creux, G.: $\quad$ vide Herzog, $\mathrm{H}$.

Deschwanden, P. von: vide Herzog, $\mathrm{H}$.

Dohi, K.: $\quad$ vide Uyeda, S.

Dufour, M. et Favez, G.:

Influence des alterations hépatiques, appréciée à Гaide d'épreuves

fonctionnelles (et accessoirement de la ponction-biopsie) sur le cours de

la tuberculose pulmonaire traitée

179

331

295121

299

417

Favez, G.:

Favez, G. Favez, G. Favez, G. Favez, G.

Guérison anatomique d'une tuberculose pulmonaire excavée durant les derniers mois d'une cachexie cancéreuse

vide Bonstein, H. vide Dufour, M. vide Ramel, C. vide Vulliémoz 117 
Favez, G. et Bonstein, H.:

Effets d'un agent tensio-actif sur la résorption de $\Gamma$ isoniazide admi-

nistré par aerosols

Friedrich, T.: vide Bonstein, $\mathrm{H}$.

Froehlich, W.: II ruolo della toracoplastica nel trattamento della tubercolosi pol-

monare

Gartmann, J.Ch. : Zur Differentialdiagnose chronischer Lungenprozesse

150

328161

Grumbach, A.:Aktuelle Sanatoriumsprobleme

Grumbach, A. und Stünzi, H.:

Die Tuberkulose bei Katze und Hund und ihre epidemiologische Be-

deutung 82

Haemmerli, U. P. und Siebenmann, R. E.:

Indikation zur Leberbiopsie bei Tuberkulose, speziell zur Frühdia-

gnose der Miliartuberkulose 297

Herzog, H.; Staub, H.; Reber, H.; Pfaltz, R.; Bertrab, R. von; Burri, H. P.;

Creux, G.; Deschwanden, P. von; Schmid, R. und Voute, C.:

Kombinierte Behandlung der Lungentuberkulose mit DSM-Panto-

thenat, INH und PAS in Dauerinfusion 303

Kirchhoff, H.W.: Über die Behandlung kindlicher Tuberkuloseformen mit Tebafen (GT3) $\quad 93$

Kneiszl, F.: Angeborene Kehlkopfatresie und beiderseitige Lungenhyperplasie . 196

Krackhardt, H.: Blutspiegel und Ausscheidung des Cycloserins bei länger dauernder

Medikation 403

Lauener, $\mathrm{H}$.: vide Bonstein, $\mathrm{H}$.

Lobenwein Weinegg, E.:

Die Pleurakaverne 267

Naef, A. P.: $\quad$ Le traitement chirurgical, par voie abdominale, de la hernie diaphragmatique 351

Ostry, P. und Baly, Z.:

Über seltene Bronchialanomalien und ein peripheres Bronchial-

adenom 392

Papp, A.: $\quad$ vide Vamos, G.

Pfaltz, R.: $\quad$ vide Herzog, $\mathrm{H}$.

Ramel, C. et Favez, G.:

Elan imprimé par les corticoïdes à la regression des cavernes tuber-

culeuses dans la période post-hormonale $\quad 373$

Reber, H.: $\quad$ vide Herzog, $\mathrm{H}$.

Regli, J.: $\quad$ Ergebnisse eines Versuches zur Förderung und Erfassung der ambu-

lanten, tuberkulostatischen Nachbehandlung von der Heilstätte aus 353

Rist, N.: $\quad$ La frequence de la resistance aux medicaments antibacillaires . . 345

Rossi, G.: $\quad$ vide Bonstein, $\mathrm{H}$.

Sauter, A.: $\quad$ Zur Frage der Verwendung der Tuberkuloseheilstätten

Schmid, R.: vide Herzog, $\mathrm{H}$. 
Schmidt, P. G.: $\quad$ Zum tuberkulösen Rundherd 250

Seiler, E.: $\quad$ Über die Epidemiologie der Sarcoidose (Morbus Boeck) in der Schweiz 205

Siebenmann, R. E.: vide Haemmerli, U. P.

Staub, ËL: vide Herzog, $H$.

Steinitz, K. H.: Weitere Erfahrungen mit D-Cycloserin in der Heilstättenbehandlung der Lungentuberkulose $\quad 330$

Steinlin, H.: Die Behandlung der Lungentuberkulose mit Ledercort in Kombination mit intensiver Chemotherapie 363

Strauss, R.: $\quad$ Komplikationen im Bereiche der unteren Luftwege beim Tauchkropf 342

Stünzi, H.: $\quad$ vide Grumbach, A.

Sturzenegger, H.: Lungenendometriose unter dem Bild des Rundschattens 259

Tatti, V.: Contribution à l'étude des effets biologiques d'un lipopolysaccharide

d'origine bactérienne dans la tuberculose pulmonaire

Uesaka, I.: vide Uyeda, I.

Uyeda, S.; Uesaka, I. et Dohi, K.:

Modification considerable de la virulence des bacilles tuberculeux

chez les souris par Гenrobage dans Thuile de paraffine $\quad 381$

Vámos, G. und Papp, A.:

Pancoast-Syndrom und Lungentuberkulose 423

Virách, Z.: $\quad$ Primary Pulmonary Torulosis 186

Voûte, C.: $\quad$ vide Herzog, $\mathrm{H}$.

Vulliémoz, P. et Favez, G.:

Le taux de properdine dans la tuberculose pulmonaire $\quad 286$

Wernli-Hässig, A.:

Zur Prognose der offenen Lungentuberkulose

229

Societates Schweizerische Vereinigung gegen die Tuberkulose. Gesellschaft

Schweizerischer Tuberkuloseärzte. 40. wissenschaftliche Tagung in

Interlaken, 7./8. Mai 1960. - Association Suisse contre la Tubercu

lose. Société des Médécins Suisses specialises en Tuberculose. 40e re

union scientifique à Interlaken, le 7/8 mai 1960293

Memorandum 92

Libri 91, 228, 290, 370, 431

Varia 160,431

Alle Rechte, insbesondere das der ÜbeГsetzung in fremde Sprachen, vorbehalten.

Ohne ausdriickliche Genehmigung des Verlages ist es auch nicht gestattet. diesen Band oder

Teile

daraus auf photomechanischem Wege (Photokopie, Mikrokopie) zu vervielfältigen.

(C)

Copyright 1960 by S. Karger AC, Basel

Printed in Switzerland

Cliches: Aberegg-Steiner \& Cie AC, Bern, und Sterner \& Cie., AC, Basel

Druck der Busier Druck- und Ver lag sans talt, Basel 